\title{
Artigos
}

\section{Supervisão acadêmica do Programa Mais Médicos na Paraíba, Brasil: percepção dos médicos brasileiros e estrangeiros}

\author{
Academic supervision of the More Doctors Program in Paraíba, Brazil: \\ Perception of Brazilian and foreign doctors (abstract: p. 18)
}

Supervisión académica del Programa Más Médicos en el Estado de Paraíba, Brasil: percepción de los médicos brasileños y extranjeros (resumen: p. 18)

\author{
Ranulfo Cardoso Junior ${ }^{(a)}$ \\ <ranulfo.cardoso@uol.com.br> \\ Eduardo Sérgio Soares Sousa(b) \\ <esergiosousa@uol.com.br>
}

\author{
(a) Unidade Acadêmica de \\ Medicina, Centro de Ciências \\ Biológicas e da Saúde, \\ Universidade Federal de \\ Campina Grande (UFCG) \\ Avenida Juvêncio Arruda, 795, \\ Bodocongó. Campina Grande, \\ PB, Brasil. 58429-600. \\ (b) Centro de Ciências \\ Médicas, Campus 1, Cidade \\ Universitária, UFPB. João \\ Pessoa, PB, Brasil.
}

Esta pesquisa avaliou a supervisão acadêmica do Programa Mais Médicos na Paraíba (PMM-PB), na perspectiva dos supervisionados, e se esta difere entre brasileiros e estrangeiros. Utilizou-se metodologia qualitativa documental e o banco de dados do inquérito conduzido sobre o PMM-PB no período de 2015 a 2016. Respostas fechadas foram analisadas pelo Qui-Quadrado e o Teste Exato de Fisher, considerando nível de significância de $5 \%(p<0,05)$. Na análise qualitativa, usou-se a técnica de análise de conteúdo. A formação acadêmica tem tempo similar entre os grupos; na pós-graduação (Saúde da Família) e na experiência de trabalho (Atenção Primária), havia mais estrangeiros do que brasileiros. A supervisão acadêmica foi avaliada como "muito positiva", destacando-se relação cordial, fácil acesso ao supervisor, suporte às decisões clínicas e envolvimento no programa; entretanto, sua atuação foi considerada insuficiente para resolver problemas de gestão.

Palavras-chave: Supervisão acadêmica. Programa Mais Médicos. Atenção Primária à Saúde.

Junior RC, Sousa ESS. Supervisão acadêmica do Programa Mais Médicos na Paraíba, Brasil: percepção dos médicos brasileiros e estrangeiros. Interface (Botucatu). 2020; 24(Supl. 1): e190487 https://doi.org/10.1590/Interface.190487 


\section{Introdução}

O Programa Mais Médicos (PMM) foi iniciado em 2013 como estratégia do Governo Federal de enfrentamento dos problemas de acesso e utilização da Atenção Primária à Saúde (APS) pela população, em regiôes brasileiras de baixa densidade de profissionais médicos ${ }^{1,2}$. Segundo o Ministério da Saúde, o PMM é um programa de provimento médico para fortalecer a Atenção Primária à Saúde a partir de três eixos: (a) infraestrutura da APS; (b) aprimoramento da atenção médica, fortalecendo a Política Nacional de Educação Permanente em Saúde, integrando ensino-serviço e aumentando vagas nos cursos de Medicina e nas residências médicas; e (c) provimento emergencial de profissionais médicos, incluindo participação de médicos estrangeiros ${ }^{1}$. Desses, a maioria é procedente de Cuba, por meio de cooperação firmada entre governo brasileiro e Organização Pan-Americana de Saúde 3 .

Para apoio às atividades dos profissionais do PMM, os Ministérios da Saúde e da Educação determinaram a implantação da supervisão acadêmica². As atribuições do supervisor acadêmico envolvem avaliar as condiçôes estruturais da unidade, o processo de trabalho e a relação do médico supervisionado com a equipe, comunidade e gestão municipal ${ }^{4}$. Na literatura da área da Saúde, pressupóe-se que o papel do supervisor envolve a promoção de conhecimentos, competências e habilidades nos cenários de trabalho, particularmente na relação com profissionais em formação ou de ingresso recente no exercício laboral. Do supervisor, é desejável que estimule a aplicação da teoria na prática, ensinando ao supervisionado a otimizar o tempo, priorizando procedimentos e atitudes para melhorar o nível de qualidade do cuidado e reconhecendo a importância ética na prática cotidiana ${ }^{5,6}$.

O objetivo geral deste estudo foi avaliar a supervisão acadêmica do PMM-PB, sob a visão dos médicos participantes brasileiros e estrangeiros.

1) Os objetivos específicos avaliaram a supervisão acadêmica, do ponto de vista dos supervisionados, levando em consideração:

a) O perfil do médico participante: características demográficas (nacionalidade, sexo, faixa etária e raça), formação acadêmica (graduação e pós-graduação) e experiência profissional.

b) Os aspectos qualitativos desta supervisão acadêmica, na visão desses supervisionados, de acordo com suas nacionalidades (brasileira ou estrangeira), no propósito de explicar se ocorrem variações entre brasileiros e estrangeiros; e como e por que estas acontecem. 


\section{Metodologia}

Este estudo utilizou metodologia qualitativa com dados secundários documentais de um banco de dados de pesquisa resultante de um inquérito previamente conduzido

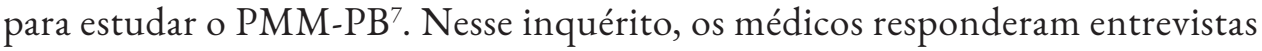
individuais com questionário semiestruturado contendo 121 questóes. Essas entrevistas foram registradas pelo médico do PMM-PB participante no estudo, e envolveram o preenchimento eletrônico pelo Google Forms de um questionário coletado no período de novembro de 2015 a março de $2016^{8}$ para formar o banco de dados da pesquisa intitulada "Análise político-social da implantação do Programa Mais Médicos no estado da Paraíba”. Este estudo obteve aprovação do Comitê de Ética em Pesquisa do Centro de Ciências da Saúde da Universidade Federal da Paraíba, em 22 de outubro de 2015, sob o número 48948015.8.0000.5188. Neste artigo, utilizaram-se os registros de 36 questôes do banco de dados original ${ }^{8}$ dos 251 médicos entrevistados que concordaram em participar da pesquisa e consentiram o uso de seus dados, que foram analisados de forma agrupada e anônima, armazenados e analisados por um sistema computadorizado, mantendo-se a confidencialidade de acordo com a legislação nacional.

Em relação ao perfil dos médicos supervisionados, foram analisadas 36 questôes relacionadas aos temas de identificação, consentimento do uso de dados, caracterização da amostra e supervisão acadêmica. Em relação à supervisão acadêmica, foram avaliadas 14 questốes das 36 selecionadas do banco de dados (questóes 100 a 113), sendo a última uma questão aberta.

Foram obtidos ainda dados sobre a avaliação geral da supervisão, participação do supervisor em reuniôes de equipe da unidade e com a gestão da saúde municipal; estratégias de educação permanente do supervisor; e avaliação/nota do supervisionado sobre a qualidade da supervisão acadêmica com escore variando de zero (péssima) a dez (excelente).

A questão aberta identificou os recursos pedagógicos que foram fornecidos pelo supervisor aos médicos do programa e, desses, quais foram considerados positivos que deveriam ser mantidos e quais os negativos e que deveriam ser suspensos ou melhorados.

$\mathrm{Na}$ análise qualitativa dos dados, usou-se a técnica de análise de conteúdo embasada

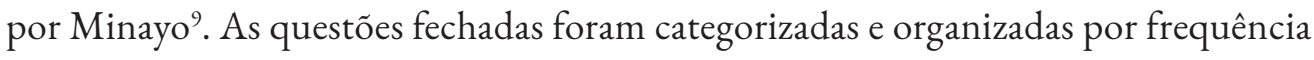
absoluta e percentual para afirmaçóes concordantes ou discordantes, utilizando-se os testes Qui-Quadrado $\left(x^{2}\right)$ e Exato de Fisher para avaliar diferenças significativas com um poder de $80 \%$ e Intervalo de Confiança (IC) de $95 \%$, considerando-se nível de significância para $\mathrm{p}<0,05$. Com relação à questão aberta, a análise de conteúdo foi temática. Das respostas elencadas pelos entrevistados, algumas foram selecionadas e agrupadas por temas. 


\section{Resultados e discussão}

\section{Seleção dos participantes da pesquisa}

Dos 294 médicos registrados no PMM-PB, 275 (93,5\%) encaminharam as respostas do questionário da pesquisa. Entre esses, 24 não consentiram usar suas informaçôes, sendo incluídos no estudo as respostas de 251 médicos e analisadas as 36 perguntas definidas na metodologia. Por questão didática, os resultados foram apresentados obedecendo aos objetivos específicos.

Inicialmente, foram caracterizados dados demográficos dos participantes do estudo quanto à nacionalidade, seguida pela avaliação da supervisão acadêmica sob influência desse aspecto.

\section{Nacionalidade de origem [subtítulo]}

$\mathrm{Na}$ caracterização do perfil de nacionalidade, de todos os 251 médicos incluídos no estudo, a maioria - 70,9\% $(\mathrm{n}=178)$ - tinha como país de origem o Brasil, e $29,1 \%(n=73)$, outros cinco países, sendo quatro latino-americanos e um europeu.

A tabela 1 mostra a distribuição dos médicos estrangeiros do programa, na Paraíba, por país de origem.

Tabela 1. Distribuição dos médicos estrangeiros do PMM-PB. Período: novembro de 2015 a março de 2016.

\begin{tabular}{lcc}
\hline País de origem & \multicolumn{2}{c}{ Médicos } \\
& $n$ & $\%$ \\
\hline Cuba & 73 & 100 \\
\hline Venezuela & 68 & 93 \\
\hline Argentina & 2 & 2,8 \\
\hline Uruguai & 1 & 1,4 \\
\hline Espanha & 1 & 1,4 \\
\hline
\end{tabular}

n: número de médicos.

\%: percentual em relação ao n.

Conforme apresentado na tabela 1 , entre os médicos estrangeiros, predominavam os cubanos (93\%) e os demais (7\%) tinham origem de quatro distintos países: Venezuela, Argentina, Uruguai e Espanha.

Comparado com o perfil dos médicos do PMM atuando no Brasil em fevereiro de 2016, quanto à nacionalidade, nossos achados mostraram que, no PMMM-PB, com relação aos médicos participantes, os estrangeiros apresentam uma relação inversa, com predomínio de quase duas vezes e meia de médicos brasileiros em relação aos estrangeiros, enquanto para todo o PMM no território nacional, os brasileiros representavam 
aproximadamente seis vezes menos comparados aos estrangeiros, que representavam $85 \%{ }^{10}$. No entanto, tanto no nosso estudo quanto no daqueles autores ${ }^{10}$, houve elevada predominância dos cubanos entre os estrangeiros, sendo 93\% cubanos nos nossos achados e $75 \%$ cubanos no estudo com dados nacionais ${ }^{10}$.

\section{Características dos médicos brasileiros e estrangeiros do PMM-PB}

\section{Distribuição geográfica versus nacionalidade}

Os resultados da Tabela 2 mostram a distribuição dos 251 médicos do PMM-PB por macrorregiáo de exercício profissional na Paraíba e nacionalidade (brasileira ou estrangeira).

Tabela 2. Distribuição dos médicos do PMM-PB incluídos no estudo por nacionalidade (brasileira ou estrangeira) nas quatro macrorregiões do estado da Paraíba. Período: novembro de 2015 a março de 2016.

\begin{tabular}{|c|c|c|c|c|c|c|c|}
\hline \multirow[b]{3}{*}{ Macrorregião } & \multicolumn{6}{|c|}{ Nacionalidade } & \multirow{4}{*}{$\mathrm{p}$-valor } \\
\hline & \multicolumn{2}{|c|}{ Todos } & \multicolumn{2}{|c|}{ Brasileira } & \multicolumn{2}{|c|}{ Estrangeira } & \\
\hline & $\mathrm{n}$ & $\%$ & $\mathrm{n}$ & $\%$ & $\mathrm{n}$ & $\%$ & \\
\hline & 251 & 100 & 178 & 100 & 73 & 100 & \\
\hline & & & & & & & $<0,0001$ \\
\hline João Pessoa & 127 & 50,6 & 104 & 58,4 & 23 & 31,5 & \\
\hline Campina Grande & 82 & 32,7 & 56 & 31,5 & 26 & 35,6 & \\
\hline Patos & 23 & 9,2 & 8 & 4,5 & 15 & 20,5 & \\
\hline Sousa & 19 & 7,5 & 10 & 5,6 & 9 & 12,4 & \\
\hline
\end{tabular}

n: número de médicos.

\%: percentual em relação ao $n$.

Conforme mostra a tabela 2, os médicos do PMM-PB estavam distribuídos em todas as macrorregióes de saúde da Paraíba, estando a maioria alocada em João Pessoa e Campina Grande, representando 50,6\% e 32,7\%, respectivamente. Assim como os brasileiros, os médicos estrangeiros se concentraram nas duas maiores macrorregióes do estado. Mais da metade dos brasileiros - 58,4\% ( $n=104)$ - foram designados para a macrorregião da capital da Paraíba e 31,5\% ( $\mathrm{n}=56)$, para Campina Grande. Poucos brasileiros foram alocados em Patos $-4,5 \%(n=8)$ - e em Sousa - 5,6\% $(n=10)$.

Similarmente aos brasileiros, os médicos estrangeiros concentraram-se nas duas maiores macrorregiôes do estado: 31,5\% ( $n=23)$ em João Pessoa e 35,6\% ( $n=26)$ em Campina Grande, embora também tenham sido designados para macrorregionais sediadas em Patos e Sousa $-20,5 \%(n=15)$ e $12,4 \%(n=9)$, respectivamente $(p<0,0001)$. 


\section{Sexo, faixa etária e raça versus nacionalidade}

A caracterização demográfica dos médicos brasileiros e estrangeiros mostrou os seguintes achados:

Sexo - A relação masculino-feminino foi de 1:1,01 para toda a amostra e, para os médicos brasileiros: $51,1 \%: 48,9 \%$, enquanto para estrangeiros essa relação foi de $43,8 \%: 56,2 \%$, respectivamente. Nos médicos brasileiros, predominavam os homens e, nos estrangeiros, as mulheres, mas sem significação estatística $(p=0,3315)$.

Faixa etária - Os médicos supervisionados apresentavam-se predominantemente mais jovens, uma vez que $45,4 \%$ desses tinham entre 25 e 35 anos, decrescendo, em número, com o aumento das faixas etárias de 35 a 45 anos, 45 a 55 anos e 55 ou mais anos, correspondendo a 22,7\%,16,3\% e 13,5\%, respectivamente. Analisados separadamente, por nacionalidade, observou-se existir diferença entre os médicos brasileiros e estrangeiros quanto ao padrão de distribuição das faixas etárias $(p<0,0001)$. Houve predominância de médicos adultos jovens entre os brasileiros, uma vez que $59,6 \%$ estão na faixa etária entre 25 e 35 anos, totalizando $77,6 \%$ abaixo de 45 anos de idade. Por outro lado, os estrangeiros concentraram-se na faixa etária de 45 a 55 anos de idade (46,6\%), seguidos de $34,2 \%$ entre 35 e 45 anos.

Outros autores mostraram médicos do PMM de todo o Brasil com perfil de idade mais elevada do que o encontrado no nosso estudo. Girardi et al. encontraram médicos do PMM com faixa etária entre 40 e 49 anos em $39,8 \%{ }^{10}$. Separadamente, os médicos brasileiros do PMM-PB se mostraram mais jovens do que os estrangeiros, tanto nos nossos achados - $54 \%$ de brasileiros entre 30 e 39 anos - quanto no estudo conduzido por Girardi et al. - 59,6\% na faixa etária entre 25 e $35 \operatorname{anos}^{10}$.

Raça - Em relação à raça, a maioria dos médicos entrevistados autodenominou-se branco ou pardo (88,5\%), e não houve diferenças aparentes entre os médicos brasileiros e os estrangeiros - 90,4\% e $83.5 \%$, respectivamente. A raça negra foi minoria entre os médicos do programa (5,2\%), com variaçóes relevantes entre as nacionalidades ( $\mathrm{p}$ $=0,0019$ ). No grupo de médicos estrangeiros, os negros eram aproximadamente seis vezes mais frequentes do que no grupo de brasileiros - 12,3 e 2,2\%, respectivamente.

\section{Formação médica - graduação versus nacionalidade}

A tabela 3 resume as características da formação médica (graduação) dos médicos do PMM-PB, distribuídos por nacionalidade (brasileira versus estrangeira), associada ao país e ao tempo gasto na formação médica na graduação. 
Tabela 3. Características da formação de graduação em Medicina da população de médicos do PMM-PB, distribuídos por nacionalidade (brasileira ou estrangeira). Período: novembro de 2015 a março de 2016.

\begin{tabular}{|c|c|c|c|c|c|c|}
\hline \multirow{4}{*}{ Formação médica } & \multicolumn{6}{|c|}{ Nacionalidade } \\
\hline & \multicolumn{2}{|c|}{ Todas } & \multicolumn{2}{|c|}{ Brasileira } & \multicolumn{2}{|c|}{ Estrangeira } \\
\hline & $\mathrm{n}$ & $\%$ & $\mathrm{n}$ & $\%$ & $\mathrm{n}$ & $\%$ \\
\hline & 251 & 100 & 178 & 70,9 & 73 & 29,1 \\
\hline \multicolumn{7}{|l|}{ País da graduação } \\
\hline Brasil & 160 & 63,7 & 160 & 89,9 & - & - \\
\hline Cuba & 79 & 31,5 & 10 & 5,6 & 69 & 94,5 \\
\hline Bolívia & 4 & 1,6 & 4 & 2,2 & - & - \\
\hline Venezuela & 3 & 1,2 & 1 & 0,6 & 2 & 2,7 \\
\hline Argentina & 2 & 0,8 & 1 & 0,6 & 1 & 1,4 \\
\hline Espanha & 2 & 0,8 & 1 & 0,6 & 1 & 1,4 \\
\hline Equador & 1 & 0,4 & 1 & 0,6 & - & - \\
\hline \multicolumn{7}{|c|}{ Duração da graduação de Medicina (anos) } \\
\hline 5 & 2 & 0,8 & 1 & 0,6 & 1 & 1,4 \\
\hline 6 & 238 & 94,8 & 171 & 96,1 & 67 & 91,8 \\
\hline 7 & 5 & 2,0 & 3 & 1,7 & 2 & 2,7 \\
\hline 8 & 2 & 0,8 & 2 & 1,1 & - & - \\
\hline Não informado & 4 & 1,6 & 1 & 0,6 & 3 & 4,1 \\
\hline
\end{tabular}

n: número de médicos PMM-PB.

\%: percentual em relação ao $\mathrm{n}$.

Os médicos do PMM-PB foram graduados em sete países, sendo que a maioria dos médicos se formou no Brasil, em primeiro lugar, e em Cuba. O Brasil predominou como país de formação da graduação médica, sendo responsável por $63,7 \%$, seguido de Cuba, 31,5\%. Juntos, esses dois países representaram 95,2\% dos centros de formação, seguidos da Venezuela, Bolívia, Argentina, Espanha e Equador.

Observa-se na tabela 3 que, quanto à duração do curso de graduação médica, a quase totalidade $(94,8 \%)$ cursou em seis anos. Os demais cursaram em sete, oito ou cinco anos $(2 \% ; 0,8 \% ; 0,8 \%)$, respectivamente. Não se evidenciaram diferenças no tempo de duração da graduação em Medicina entre médicos brasileiros e estrangeiros.

O Brasil vem apresentando aumento progressivo de escolas médicas. De acordo com a publicação Demografia Médica (2015), os números dessas escolas no país eram 77, em 1985; 82, em 1990; e 247, em 2014¹1. Em 1996, o Brasil contava com 84 escolas médicas, majoritariamente públicas, e o estado da Paraíba continuava com suas duas escolas médicas federais e a única do interior no Nordeste, em Campina Grande ${ }^{12}$. No ano de 2019, a Paraíba possuía nove escolas médicas, sendo três públicas e seis privadas, ofertando 975 vagas anuais, distribuídas nas quatro macrorregióes do estado ${ }^{11,13}$. 
Considerando as evidências obtidas sobre a Paraíba em relação à sua capacidade instalada para a formação de médicos, distribuição espacial das escolas médicas no estado $^{13}$ e exigências curriculares do Ministério da Educação para essas escolas em relação ao enfoque acadêmico na Atenção Primária ${ }^{14}$, não se justifica na Paraíba o recrutamento e contratação de médicos formados em outros países para ofertar assistência médica adequada à exigida na Atenção Primária, desde que exista uma política acadêmica para formação desses profissionais nesta área de atuação.

\section{Formação médica - pós-graduação versus nacionalidade}

Dos 251 médicos participantes do estudo, a maioria (71,3\%) não apresentava formação de pós-graduação na área de Saúde da Família. Na pós-graduação em Medicina lato sensu, 49,4\% desses profissionais apresentaram formação de especialista na área da Atenção Primária (Saúde da Família), seja residência ou título de especialista. Por nacionalidade, identificou-se considerável variação dessa formação, com os estrangeiros apresentando percentual muito elevado nessa área da Atenção Primária $(93,1 \%, \mathrm{p}<0,0001)$ em comparação com apenas 2,3\% entre os médicos brasileiros que informaram possuir residência médica em Saúde da Família (ou similar, no território estrangeiro).

Metade dos médicos do PMM-PB referiu ter especialização em Saúde da Família pela Universidade Aberta do Sistema Único de Saúde (SUS); no entanto, 78,1\% dos médicos estrangeiros informaram ter essa especialização e só metade desse percentual $(37,6 \%)$ foi informado pelos brasileiros. Dessa forma, para os brasileiros, a Universidade Aberta do SUS foi a principal instituição formadora da pós-graduação lato sensu, tanto na quantidade de médicos formados quanto na área de suas atuaçóes - APS, porém, foi uma especialidade que apareceu em um percentual muito aquém do esperado. Destaca-se a determinação da obrigatoriedade dessa formação no primeiro ano do $\mathrm{PMM}^{15}$. Foi desprezível a formação em residência médica em outra área: 6,7\% ( $n=12)$, entre os brasileiros, e 19,2\% $(n=14)$, entre os estrangeiros, cerca de três vezes mais que os brasileiros, portanto.

Em relação à formação de pós-graduação em Medicina stricto sensu, dos médicos do PMM-PB, 8\% declararam possuir formação de mestrado, sendo inexistente a formação de doutorado.

Quando analisada a pós-graduação stricto sensu, por nacionalidade, informaram ter título de mestre $1,7 \%(n=3)$ e $23,3 \%(n=17)$ dos médicos brasileiros e estrangeiros, respectivamente $(\mathrm{p}<0,0001)$. Dessa forma, $85 \%$ dos títulos de mestres eram de estrangeiros. Esse resultado encontra coerência quando se observa a fragilidade na área da Saúde em relação à existência de cursos em pós-graduação nessa área de conhecimento, em discordância com o prestígio alcançado pela Universidade Federal da Paraíba e Universidade Federal de Campina Grande nas áreas tecnológicas, como atesta a Coordenação de Aperfeiçoamento de Pessoal de Nível Superior, vinculada ao Ministério da Educação por meio da Plataforma Sucupira ${ }^{16}$. 
(e)

$\mathrm{Na}$ distribuição dos cursos de pós-graduação, observa-se que a Universidade Federal da Paraíba oferece 93 cursos e a Universidade Federal de Campina Grande, 38 cursos. Em contraste, nenhuma política de fortalecimento da área da Saúde, no nível da pósgraduação, é visualizada para as escolas de Medicina das duas universidades federais.

Considerando o encontrado neste trabalho, podemos constatar que existe uma grande lacuna na formação médica em pós-graduação stricto sensu na Paraíba. Temos, assim, necessidade de uma mobilização do meio acadêmico para provocar o nível estratégico das universidades federais da Paraíba com escolas médicas vinculadas para elaborar e implantar um plano de desenvolvimento e inovação, com o propósito de equiparar os cursos de Medicina dessas universidades aos demais cursos por meio da institucionalização da pesquisa acadêmica na universidade. $\mathrm{O}$ médico e pesquisador Carlos Chagas Filho resume a relevância dessa estratégia: "Na universidade se ensina porque se pesquisa" ${ }^{\prime 1}$.

\section{Capacidade instalada de formação médica na graduação e seu contraste com a pós-graduação lato e stricto sensu: desafios da Paraíba}

Analisando os dados da demografia médica publicados em 2018, observa-se que a Paraíba contribui com 975 vagas, sendo $27,2 \%$ públicas $(n=265)$ e 72,8 privadas $(\mathrm{n}=710)$. Depois do Tocantins, na região Norte, a Paraíba aparece como o estado com o maior número de vagas por cem mil habitantes, sendo da ordem de 24,2 vagas, enquanto o Brasil apresenta 14,1 vagas $^{18}$. Esses indicadores caracterizam a Paraíba como sendo um estado com uma elevada capacidade instalada para formar médicos no país. Assim, parece haver um potencial excedente para as necessidades de formação de médicos direcionados para atuar e se fixar no próprio estado. Essa condição pode ser considerada uma oportunidade para impulsionar a Paraíba para uma política de estado de tornar-se um grande polo de ciência e tecnologia, agregando a área da Saúde à consolidada tradição da Universidade Federal de Campina Grande e da Universidade Federal da Paraíba em pós-graduação stricto sensu.

\section{Experiência profissional prévia na Atenção Primária, anterior à vinculação ao PMM-PB}

A tabela 4 mostra a experiência prévia como médico na Atenção Primária antes de ingressar no PMM-PB, por nacionalidade (brasileira ou estrangeira). 
Tabela 4. Características da população de médicos do PMM-PB, distribuídos por nacionalidade (brasileira ou estrangeira), associada à experiência profissional como médico na APS antes de ingressar no PMM-PB. Período: novembro de 2015 a março de 2016.

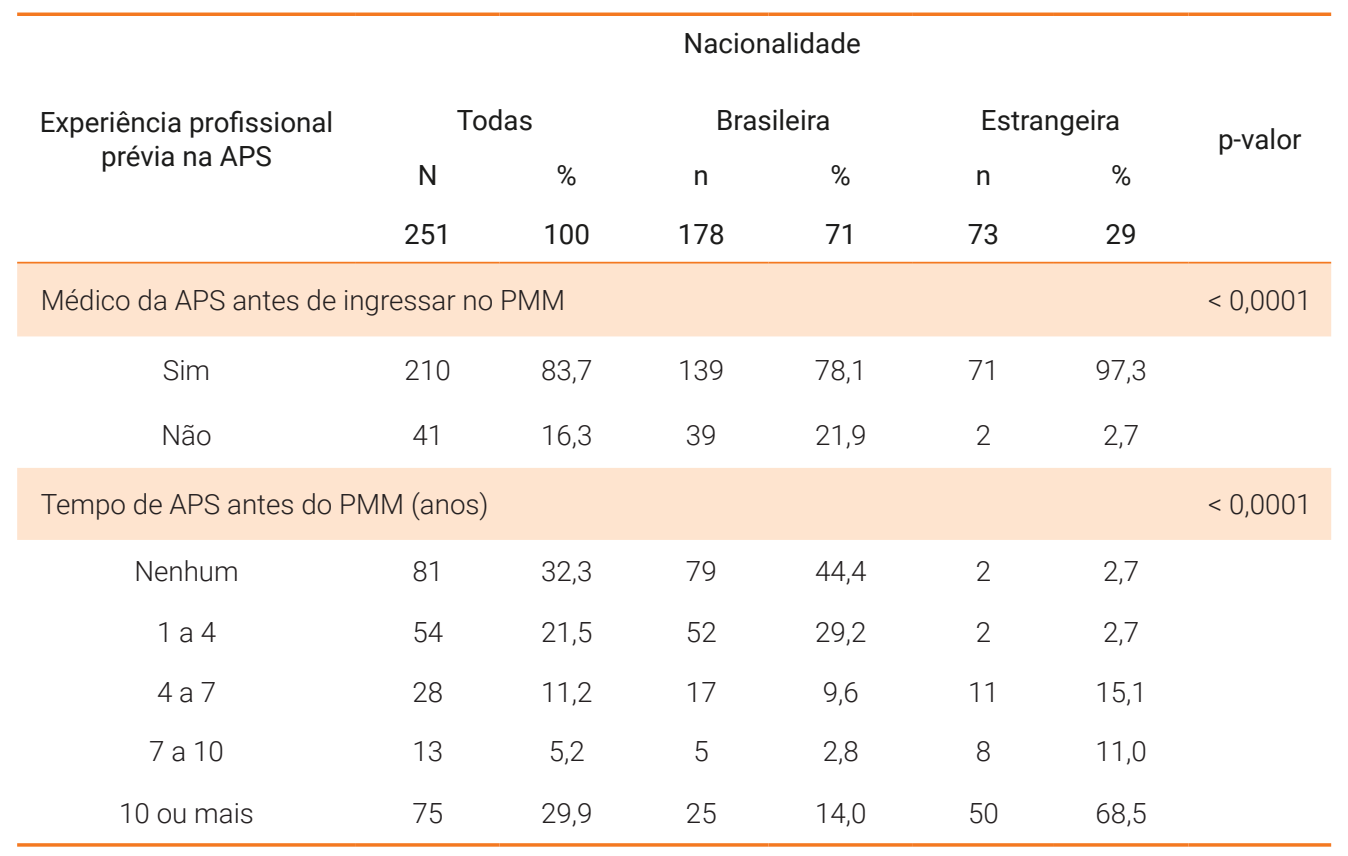

n: número de médicos PMM-PB.

\%: percentual em relação ao $\mathrm{n}$.

Com relação à experiência profissional anterior ao PMM, a maioria dos participantes (87,3\%) informou ter tido experiência de médico na APS antes de ingressar no programa. Quanto à nacionalidade, os estrangeiros apresentavam aproximadamente 20\% mais experiência prévia na APS em comparação com os brasileiros.

Ao se avaliar o tempo de experiência na Atenção Primária antes do programa, observa-se uma distribuição de frequências bimodal, com concentração nos extremos (a) nenhuma e entre um a quatro anos em $32,3 \%$ e 21,5\%, respectivamente; e (b) dez anos ou mais anos, em $29,9 \%$. Separadamente, a nacionalidade influencia o tempo de experiência prévio. A maioria dos estrangeiros (68,5\%) apresentou dez anos ou mais de experiência; em contraste, a maioria dos brasileiros não tinha nenhum ano de experiência prévia (44,4\%) ou menos de 4 anos (29,9\%).

Com relação ao tempo de formado, para todo o conjunto predomina dez ou mais anos em $41 \%$ desses médicos, seguidos por aqueles entre quatro e seis anos (39\%) e um menor percentual entre seis a oito anos (12\%). Todos os estrangeiros apresentavam tempo de formado de seis ou mais anos. Observam-se diferenças quando o tempo de formado é analisado por nacionalidade. Entre os brasileiros, percentualmente, predominavam dois períodos: um maior, entre quatro e seis anos de formado $(51,1 \%)$, e outro com dez ou mais anos de formado (21,3\%). Entre os estrangeiros, nenhum dos médicos tinha menos de seis anos de formado e a proporção daqueles com dez ou mais anos de formado mostrou-se extremamente elevada (89\%). 
O ensino em serviço de preceptoria em residência (Medicina de Família e Comunidade ou Medicina Preventiva e Social) foi referido pela maioria dos médicos estrangeiros $(58,9 \%)$ que afirmaram ter essa experiência. Em contraste, essa preceptoria era praticamente inexistente entre os brasileiros (inferior a $2 \%$ ).

O tempo no programa encontrava-se, predominantemente, no intervalo entre três e cinco anos - $51 \%$ e $26,3 \%$, respectivamente -, estando a maioria dos brasileiros há três anos no programa $(66,9 \%)$ e, dos estrangeiros, cinco anos (57,5\%). Assim, o programa não se caracterizou como uma opção de primeiro emprego. Iniciaram suas atividades profissionais, como médicos no PMM, $12 \%$ da população estudada. Por nacionalidade, esse percentual foi de $16,3 \%$ entre os brasileiros e apenas de 1,4\% entre os estrangeiros. Em relação ao tempo de atuação como médico no programa, a grande maioria dos brasileiros $(85,5 \%)$ apresentava tempo inferior a quatro anos. Diferentemente desses, $87,6 \%$ dos estrangeiros atuavam como médico do programa há quatro ou mais anos.

Rech et al. ${ }^{19}$ avaliaram a qualidade da APS em associação com o PMM no Brasil, na perspectiva dos médicos brasileiros do programa, estrangeiros do programa e brasileiros não vinculados ao Programa; e observaram ter havido um pequeno aumento no escore geral da APS, com discreta melhora. Contudo, esses autores não encontraram influência da nacionalidade na magnitude desse efeito ${ }^{19}$.

\section{Avaliação da supervisão acadêmica}

Com relação à atuação do supervisor na Unidade Básica de Saúde, 60,6\% dos médicos referiram que o número de visitas ficou entre três e quatro nos últimos três meses, seguida por uma a duas visitas $(28,3 \%)$ e cinco a seis $(9,6 \%)$. Quando o número de visitas foi relacionado à nacionalidade, tanto os brasileiros $(57,3 \%)$ quanto os estrangeiros $(68,5 \%)$ sinalizaram três a quatro visitas; uma a duas visitas foram referidas em segundo lugar, sendo em 31,4\% dos brasileiros e em 20,6\% dos estrangeiros. Relataram ainda cinco a seis visitas em $10,1 \%$ e $20,6 \%$ e nenhuma visita $1,1 \%$ e 2,7\% os médicos brasileiros e estrangeiros, respectivamente.

Quanto à participação do supervisor em alguma reunião de equipe na Unidade Básica de Saúde, foi informada afirmativamente em 49,4\% ( $\mathrm{n}=124)$ e negativamente em $50,6 \%(\mathrm{n}=127)$ dos médicos. Quando esse parâmetro foi relacionado à nacionalidade, responderam "sim" 46,1\% e 57,5\% dos brasileiros e estrangeiros, respectivamente, e responderam "não" 53,9\% e 42,5\% dos brasileiros e estrangeiros, respectivamente.

Com relação à realização de reuniões regulares com a gestão do município para discutir os problemas na APS, $87,6 \%$ dos médicos informaram que realizam. Porcentagens igualmente elevadas foram obtidas entre médicos brasileiros $(87,1 \%)$ e estrangeiros (89\%).

Este estudo mostra uma elevada satisfação dos médicos tanto brasileiros quanto estrangeiros supervisionados com o trabalho de supervisão acadêmica do PMMPB. De forma geral, há predominância dos conceitos "muito bom” e "ótimo" entre os médicos brasileiros e estrangeiros. Quase todos os estrangeiros avaliaram a supervisão com o conceito máximo.

Outra atividade de supervisão acadêmica percebida como igualmente elevada entre brasileiros e estrangeiros foi a reunião com a gestão do município $(87,6 \%)$. 


\section{Recursos metodológicos utilizados na supervisão acadêmica}

Observou-se que o uso intensivo de telefone celular, tanto por grupos de WhatsApp quanto contatos particulares, destaca-se como o instrumento para a informação e comunicação entre médicos e seus supervisores. De forma similar, os brasileiros e estrangeiros referem que os supervisores disponibilizaram, sem restriçôes, o uso de WhatsApp, e-mails e telefone celular como táticas de apoio à supervisão acadêmica.

A tecnologia da informação e comunicação de apoio à APS oferecida pela Telessaúde, entretanto, foi pouco utilizada pelo PMM-PB, sendo similares as avaliações de tal estratégia entre brasileiros e estrangeiros, uma vez que $61,8 \%$ e $67,1 \%$ deles, respectivamente, informaram que esta não foi utilizada como recurso metodológico nas visitas de supervisão.

\section{Recursos metodológicos utilizados e desejáveis}

Em relação às estratégias metodológicas de supervisão, a maioria dos médicos brasileiros pesquisados referiu que a discussão de casos foi a estratégia pedagógica mais utilizada, seguida pelo feedback do supervisor dos seus atendimentos no fim das consultas. Entre os estrangeiros, as metodologias mais mencionadas foram a discussão de temas com leitura prévia e a discussão de casos.

O apoio à Atenção Primária oferecida pela Telessaúde foi pouco utilizado pelo programa, conforme avaliaçóes similares de tal instrumento pedagógico por brasileiros e estrangeiros ${ }^{20}$. Entretanto, destacou-se como a metodologia mais solicitada, estando entre as 4 mais citadas em ambos os grupos. Esse achado é corroborado pelo estudo brasileiro sobre uso da Telessaúde no SUS, que mostra a utilização da teleconsulta pelo estado da Paraíba de apenas 2,2\% do total das realizadas no país ${ }^{20}$.

A revisão da literatura revela críticas controversas à atuação da supervisão acadêmica no PMM. Enquanto Campos et al. ${ }^{22}$ afirmam que "o sistema de supervisão e de tutoria se dirigiu apenas aos médicos e não a toda equipe em que estes médicos estão inseridos” (p. 2661), outra autora, revela - em artigo sobre intervençóes na saúde do idoso no PMM - que a supervisão, para além do médico, apoiou também a equipe multidisciplinar da Unidade Básica de Saúde e a própria gestão municipal ${ }^{21,22}$.

Há que se ponderar que, provavelmente, vivenciamos distintas atuaçóes da supervisão acadêmica em diferentes territórios do país, o que corrobora a percepção de Engstrom et al. ${ }^{23}$, que apontaram - refletindo a partir das necessidades educacionais para lidar com a gama de práticas da APS, focada em uma experiência de supervisão acadêmica do Programa Mais Médicos, no município de Niterói, Rio de Janeiro, entre 2014 e 2015 - que, em publicaçôes específicas e materiais técnicos produzidos acerca do programa, percebeu-se maior ênfase às atribuiçốes operacionais na atuação do supervisor acadêmico e identificaram-se lacunas em estratégias de trabalho e sugestões metodológicas, sobretudo no contexto da educação em saúde ${ }^{23}$. 


\section{Análise e discussão sobre a questão aberta}

$\mathrm{Na}$ exploração da questão aberta, as respostas foram lidas e pré-analisadas quanto aos conteúdos para identificação dos temas citados pelos entrevistados. Dois temas destacaram-se pelo conteúdo e frequência de citações, emergindo como positivas as atitudes dos supervisores no apoio acadêmico e como negativa a insatisfação da ação de intermediação do supervisor frente aos problemas da gestão municipal.

\section{Conteúdo positivo}

A expressiva maioria dos informantes ressaltou muito os aspectos positivos que devem ser mantidos no trabalho da supervisão acadêmica, realçando o compromisso ético-pedagógico dos supervisores, o compartilhamento da experiência acumulada destes, a acessibilidade e o bom acolhimento para dirimir dúvidas nos processos de diagnóstico e tratamento clínico, quer presencialmente ou a distância (por telefone, e-mails ou WhatsApp). Destacamos algumas falas dos supervisionados, identificados com pseudônimos, que explicitam essas consideraçóes:

No momento, eu não saberia dizer pontos negativos. Houve facilidade no diálogo, boa receptividade e o supervisor foi muito prestativo quanto a tirar dúvidas ou a esclarecer casos do nosso dia a dia. (Paula Madruga de Sá)

Recebemos sugestôes de ideias para melhorar o trabalho individual e coletivo e estímulo para escutar a população local, sobre como ela avalia o trabalho da equipe de saúde. (Ariosvaldo Medeiros Lima)

Além disso, destacou-se o engajamento do supervisor nos processos de trabalho da equipe multidisciplinar e a atitude da supervisão em relação aos gestores, habitualmente advogando pelo respeito ao trabalho dos supervisionados na busca de benefícios para as Unidades Básicas e população assistida. Tais achados são corroborados em estudos de caso sobre o PMM no estado do Rio Grande do Norte, indicando que a inserção da supervisão acadêmica nos territórios de saúde perpassa pela lógica da necessidade de ir "além dos vazios assistenciais", pois possibilita transformar as práticas de gestão do cuidado e do processo de trabalho, gerando uma nova cultura de qualificação da APS; promovendo a interação de saberes e práticas para gestão do cuidado; e incorporando promoção da saúde e prevenção de agravos ${ }^{24}$. 


\section{Conteúdo negativo ou pedidos de melhorias na supervisão}

Em relação aos pontos negativos e que precisariam ser modificados, há relatos que consideram o tempo da visita presencial reduzido. Alguns entrevistados sugeriram que a visita de supervisão fosse realizada preferencialmente no turno da tarde (face à demanda de usuários ser menor nas unidades); outros sugeriram viabilizar a interrupção dos atendimentos, nos momentos da visita do supervisor, o que possibilitaria um maior aprofundamento dos temas da agenda de supervisão acadêmica e ampliaria a integração da equipe multidisciplinar às orientaçóes dos supervisores. Houve referências, isoladas, criticando algumas visitas apressadas e, em outros casos, reclamando maior ênfase às discussóes sobre casos clínicos no cotidiano de atendimento dos médicos. A maior cobrança foi sobre o enfrentamento dos supervisores em relação a determinados aspectos administrativos e omissões da gestão da saúde municipal, tema negativo mais citado no conteúdo das narrativas.

A segunda maior reivindicação foi a discussão de artigos previamente selecionados para os brasileiros. Para os estrangeiros, o segundo instrumento pedagógico mais requerido foi a discussão de casos. Mesmo referido como utilizado, houve demanda expressiva ratificando a importância de intensificar o uso desse instrumento pedagógico.

Os temas emergentes sobre a supervisão acadêmica do PMM-PB foram organizados e categorizados para análise. Na perspectiva dos supervisionados, a supervisão acadêmica atendeu às suas expectativas nas atividades de suporte às decisões clínicas, mas foi limitada para os problemas relacionados à gestão: nossos achados de reconhecida insatisfação com a atividade de supervisão, no enfrentamento de problemas relacionados à gestão nas unidades, estão em consonância com o ensaio publicado por Campos et al. ${ }^{22}$. Esses autores analisaram as políticas da APS no Brasil, destacando a Estratégia Saúde da Família e o PMM, e consideraram, entre os principais obstáculos, questôes estruturais de financiamento insuficiente e de gestão ineficiente ${ }^{22}$.

\section{Conclusão}

Neste artigo, descreve-se o estudo sobre a supervisão acadêmica do PMM-PB na perspectiva dos médicos desse programa. Os resultados nos permitiram obter as seguintes conclusôes: de acordo com a nacionalidade - médicos brasileiros tinham faixa etária mais jovem, eram menos experientes em APS e praticamente sem formação em pós-graduação stricto sensu (mestrado) quando comparados aos médicos estrangeiros.

Em relação à percepção sobre a supervisão acadêmica, ambos, brasileiros e estrangeiros, pontuam essa atividade nos escores máximos. Nas estratégias metodológicas da supervisão, são reforçadas a elevada relevância das discussóes de casos e leitura prévia com discussão posterior.

Dentre as metodologias e técnicas pedagógicas pouco utilizadas pelos supervisores e extremamente recomendadas pelos médicos supervisionados, destacou-se o uso do sistema de informação e comunicação da Telessaúde, tanto pelos brasileiros quanto pelos estrangeiros. Da questão aberta emergiu, como recorrente, insatisfação com 
as açôes de mediação dos supervisores com problemas identificados pelos médicos relacionados à gestão das unidades de APS pela falta de infraestrutura ou insumos, competências do gestor municipal.

Com este trabalho, esperamos não só contribuir para a melhoria da supervisão acadêmica do PMM-PB, mas também provocar uma reflexão sobre as lacunas encontradas na formação de pós-graduação, entre essas, a ausência de estrutura formadora de mestres e doutores em Medicina como consequência direta da ausência de pesquisa. Os achados deste estudo mostram uma enorme carência de programas de pós-graduação lato e stricto sensu voltados para a área médica, inclusive para a APS, na Paraíba. Esforços adicionais devem ser dispensados pela Universidade Federal da Paraíba e Universidade Federal de Campina Grande para que seus cursos médicos recebam o suporte estratégico necessário, ultrapassem seu atual e único objetivo, que é a formação acadêmica de médicos, e alcancem destaque na produção de conhecimento, similarmente ao que essas instituições possuem em outras áreas.

\section{Contribuições dos autores}

Ambos os autores participaram ativamente de todas as etapas de elaboração do manuscrito.

\section{Agradecimentos}

À pesquisadora e médica Taciana Padilha de Castro, Fiocruz-PE; e à professora, pesquisadora e médica Denia Fittipaldi, da Universidade Federal de Pernambuco, a admiração e gratidão dos autores, pelo estímulo e disponibilidade para a reflexão crítica durante todo o processo que culminou com a publicação do presente artigo.

\section{Direitos autorais}

Este artigo está licenciado sob a Licença Internacional Creative Commons 4.0, tipo BY (https://creativecommons.org/licenses/by/4.0/deed.pt_BR).

\section{(cc)BY}




\section{Referências}

1. Brasil. Ministério da Saúde. Lei no 12.871, de 22 de Outubro de 2013. Institui o Programa Mais Médicos, altera as Leis no 8.745, de 9 de Dezembro de 1993, e no 6.932, de 7 de Julho de 1981, e dá outras providências. Diário Oficial da União. 23 Out 2013.

2. Brasil. Ministério da Saúde. Ministério da Educação. Portaria Interministerial no 1.369, de 8 de julho de 2013. Dispóe sobre a implementação do Projeto Mais Médicos para o Brasil. Diário Oficial da União. 9 Jul 2013.

3. Brasil. Ministério da Saúde. Terceiro Termo de Ajuste ao $80^{\circ}$ Termo de Cooperação Técnica para o desenvolvimento de açóes vinculadas ao Projeto "Acesso da População Brasileira à Atenção Básica em Saúde”, que entre si celebram a união, por intermédio do Ministério da Saúde/Fundo Nacional de Saúde, e a Organização Pan-Americana de Saúde/Organização Mundial de Saúde. Diário Oficial da União. 22 Ago 2013.

4. Brasil. Ministério da Educação. Portaria Normativa no 14, de 9 de Julho de 2013. Dispóe sobre os procedimentos de adesão das Instituiçóes Federais de Educação Superior ao Projeto Mais Médicos e dá outras providências. Ministério da Saúde. Diário Oficial da União. 10 Jul 2013; sec. 1, no 131, p. 18.

5. Brasil. Ministério da Educação. Resolução CNRM no 005, de 6 de Junho de 2004 . Dispóe sobre os serviços de preceptor/tutor dos programas de Residência Médica. Diário Oficial da União. 11 Jun 2004; sec. 1, p. 19.

6. Borges MC, Frezza G, Souza CS, Bollela VR. Ensino clínico em cenários reais de prática. Medicina (Ribeirão Preto). 2015; 48(3):249-56. Doi: http://dx.doi. org/10.11606/issn.2176-7262.v48i3p249-256.

7. Almeida ER, Martins AF, Macedo HM, Penha RC. Projeto Mais Médicos para o Brasil: uma análise da supervisão acadêmica. Interface (Botucatu). 2017; 21 Supl 1:1291-300. Doi: http://dx.doi.org/10.1590/1807-57622016.0558.

8. Sassi AP. As instituiçóes de ensino no Programa Mais Médicos: tutoria e supervisão [tese]. João Pessoa: Universidade Federal da Paraíba; 2018.

9. Minayo MCS. O desafio do conhecimento: pesquisa qualitativa em saúde. São Paulo: Hucitec; 2014.

10. Girardi SN, Carvalho CL, Pierantoni CR, Costa JO, Stralen ACSV, Lauar TV, et al. Avaliação do escopo de prática de médicos participantes do Programa Mais Médicos e fatores associados. Cienc Saude Colet. 2016; 21(9):2739-48. Doi: https://doi. org/10.1590/1413-81232015219.15912016.

11. Scheffer M, Biancarelli AC, Cassenote A. Demografia médica no Brasil 2015. São Paulo: Faculdade de Medicina da USP, Conselho Regional de Medicina do Estado de São Paulo, Conselho Federal de Medicina; 2015.

12. Amaral JA. Duzentos anos de ensino médico no Brasil [tese]. Rio de Janeiro: Universidade do Estado do Rio de Janeiro; 2007.

13. Nassif ACN. Escolas Médicas por Estado [Internet]. 2019 [citado 20 Jan 2019]. Disponível em: https://www.escolasmedicas.com.br/escolas-medicas-estado.php

14. Moreira COF, Dias MSA. Diretrizes curriculares na saúde e as mudanças nos modelos de saúde e de educação. ABCS Health Sci. 2015; 40(3):300-5. Doi: http://dx.doi. org/10.7322/abcshs.v40i3.811.

15. Campos FE, Lemos AF, Vianna RF, Oliveira VA, Franco SM, Nascimento EM, et al. Experiências exitosas da rede UMA-SUS: trajetórias de fortalecimento e consolidação da Educação Permanente em Saúde no Brasil. São Luís: EDUFMA; 2017. 
16. Plataforma-Sucupira. Cursos avaliados e reconhecidos [Internet]. Brasíla; 2019 [citado 20 Jan 2019]. Disponível em: https://sucupira.capes.gov.br/sucupira

17. Instituto de Biofísica Carlos Chagas Filho. O Instituto de Biofísica Carlos Chagas Filho [Internet]. Rio de Janeiro; 2019 [citado 21 Jan 2019]. Disponível em: https:// www.biof.ufrj.br/

18. Scheffer M, Cassenote A, Guilloux AGA, Miotto BA, Mainardi GM. Demografia médica no Brasil 2018. São Paulo: Faculdade de Medicina da USP, Cremesp; 2018.

19. Rech MRA, Hauser L, Wollmann L, Roman R, Mengue SS, Kemper ES, et al. Qualidade da Atenção Primária à saúde no Brasil e associação com o Programa Mais Médicos. Rev Panam Salud Publica. 2018; 42:e164. Doi: https://doi.org/10.26633/ RPSP.2018.164.

20. Almeida BLJ. Telessaúde Brasil Redes Conectando o SUS. Rev Bras Med Fam Comunidade. 2014; 15:64-7.

21. Bertão IR. A atuação de um médico do Programa Mais Médicos para o Brasil e a mudança processo de trabalho da equipe de Estratégia de Saúde da Família. Tempus (Brasília). 2015; 9(4):217-24. Doi: http://dx.doi.org/10.18569/tempus.v9i4.1739.

22. Campos GWS, Pereira JRN. A Atenção Primária e o Programa Mais Médicos do Sistema Único de Saúde: conquistas e limites. Cienc Saude Colet. 2016; 21(9):2655-63.

23. Engstrom EM, Carvalho LC, Romano VF, Castro AAC. O supervisor e as estratégias educacionais dos encontros locorregionais no programa Mais Médicos do Brasil: reflexões acerca de concepções e práticas. Tempus (Brasília). 2016; 10(1):241-52. Doi: http://dx.doi.org/10.18569/tempus.v10i1.1863.

24. Organização Pan-Americana de Saúde. Estudo de caso do Programa Mais Médicos no Rio Grande do Norte: caminhos percorridos, produções e criações de conhecimento no Semiárido Potiguar. Brasília: OPAS; 2016. 
This study assessed the academic supervision of the More Doctors Program in the State of Paraíba, Brazil, in the perspective of the supervisees, and analyzed whether it differs among Brazilians and foreigners. The documental qualitative methodology was used, as well as the database of the inquiry conducted about the More Doctors Program-Paraíba from 2015 to 2016. Closed-ended answers were analyzed by the chi-square test and by Fisher's Exact Test, considering a level of significance of $5 \%(\mathrm{p}<0.05)$. In the qualitative analysis, the content analysis technique was used. The two groups have a similar academic education in terms of time; in postgraduate education (family health), foreigners outdid Brazilians; the same happened concerning work experience (primary care). Academic supervision was considered "very positive" and the main reasons for this evaluation were cordial relationship, easy access to the supervisor, support to clinical decisions, and involvement in the Program; however, the supervisors' action was considered insufficient to solve management problems.

Keywords: Academic supervision. More Doctors Program in Paraíba. Primary Health Care.

Este estudio evaluó la supervisión académica del Programa Más Médicos, en el Estado de Paraíba, bajo la perspectiva de los supervisados y si ella difiere entre brasileños y extranjeros. Se utilizó la metodología cualitativa documental y el Banco de Datos del cuestionario realizado sobre el Programa Más Médicos -Estado de Paraíba, período 2015 a 2016. Las respuestas cerradas se analizaron por el Chi cuadrado y el Test Exacto de Fischer, considerado nivel de significancia del $5 \%(\mathrm{p}<0,05)$. En el análisis cualitativo se utilizó la técnica de análisis de contenido. La formación académica tiene tiempo similar entre los grupos; en el postgrado (salud de la familia) y en experiencia de trabajo (atención primaria), los extranjeros superaron a los brasileños. La supervisión académica se evaluó como “muy positiva”, destacándose la relación cordial, el fácil acceso al supervisor, el soporte a las decisiones clínicas y el envolvimiento en el Programa; no obstante, se consideró insuficiente su actuación para resolver problemas de gestión.

Palabras clave: Supervisión académica. Programa Más Médicos en el Estado de Paraíba. Atención Primaria de la Salud. 